\title{
The rhythm span task: Comparing memory capacity for musical rhythms in musicians and non-musicians
}

\author{
Nora K. Schaal $^{1}$, Michael J. Banissy ${ }^{2} \&$ Kathrin Lange $^{1}$ \\ 1. Institut für Experimentelle Psychologie, Heinrich-Hein-Universität, Düsseldorf, Germany \\ 2. Department of Psychology, Goldsmiths, University of London, London, UK
}

\author{
Corresponding author: \\ Nora K. Schaal \\ Heinrich-Heine-Universität Düsseldorf \\ Institut der Experimentellen Psychologie \\ Universitätsstraße 1 \\ 40225 Düsseldorf \\ Telefone: +492118113 \\ Fax: +49-211-811-3490 \\ Email:nora.schaal@uni-duesseldorf.de
}

\begin{abstract}
The study introduces a rhythm memory task and compares performance of musicians and non-musicians. The rhythm span task with increasing and decreasing sequence length according to the participants' performance measures the individual memory capacity for musical rhythms. Results show that musicians perform significantly better on the rhythm span task indicating that memory capacity for rhythms is superior after many years of formal musical training. Additionally performance correlates positively with an established pitch span task (Williamson \& Stewart, 2010) as well as the five dimensions of the Gold-MSI selfreport questionnaire (Müllensiefen et al., 2014) evaluating musical sophistication.
\end{abstract}

Running Head: The Rhythm Span Task 


\section{Introduction}

Rhythm is one of the main basic building blocks in music cognition (Krumhansl, 2000) and an important element of spoken language and language perception (Nazzi, Bertoncini \& Mehler, 1998; Ramus, Nespor \& Mehler, 1999). In music rhythm is defined as the temporal organization of the musical material classified by the onset and duration of stimuli and the intervals between the onsets (Krumhansl, 2000; Grahn, 2012). Rhythm perception has been studied with growing interest behaviourally (e.g. Fischinger, 2011; Grahn \& Rowe, 2009; McAuley \& Henry, 2010) and in neuropsychological studies (see Grahn, 2012 for an overview) but research in the memory process of rhythmical elements has not found yet much attention in the literature.

Research groups looking at rhythm perception have developed several models regarding the organization of rhythmical sequences (e.g. the temporal grid model by Povel, 1984; the internal clock by Povel \& Essens, 1985; in the Generative Theory of Tonal Music by Lerdahl \& Jackendorff, 1983). Many studies have examined the hierarchical structures of rhythm sequences and found that several factors influence rhythm reproduction (Drake, 1993; Drake, Penel \& Bigand, 2000; Wu et al., 2013). For example, Drake (1993) showed that binary rhythms (compared to ternary subdivision), two different durations in one sequence (by contrast to three durations), accents on important beats and the possibility to segment into shorter sequences, lead to better performance for rhythm reproduction.

Rammsayer \& Altenmüller (2006) compared performance of musicians and nonmusicians on perceptual auditory temporal tasks and revealed superior performance of the musicians group on rhythm perception, auditory fusion and temporal discrimination tasks. By contrast, no significant difference in a temporal generalization task was found between the two groups. The authors relate this to the fact that the generalization task requires the storage of the reference interval in long-term memory: Because the underlying process is not automatic they assume that temporal judgment is less sensitive to musical training (Rammsayer \& Altenmüller, 2006). However other studies have shown superior performance of musicians in memory tasks (e.g. Wallentin et al., 2010). Furthermore, studies have shown superior temporal accuracy of musicians on perceptual rhythm tasks (Geiser et al., 2009; Jones and Yee, 1997; Rammsayer, Buttkes \& Altenmüller, 2012) indicating that musicians, as experts in the domain, have developed superior skills in rhythm perception.

The literature including rhythm memory tasks is to our best knowledge relatively sparse. As part of the Musical Ear Test (Wallentin et al., 2010) a rhythm memory task was 
introduced where subjects are required to make same/different judgments after hearing two rhythm sequences. Interestingly, there is empirical evidence showing that the rhythm memory task can be used to distinguish groups according to musical training and ability as musicians scored significantly higher on both parts of the test (rhythm and pitch memory) (Wallentin et al., 2010). In another study, Saito (2001) used a rhythm memory task in which non-musicians heard rhythmical sequences and were required to memorize and reproduce them by tapping the pattern on a computer key. Results show that performance on this rhythm memory and reproduction task correlated with an aurally presented digit span task which indicates that superior performance of rhythm memory is linked to better memory in the auditory domain in general.

Functional imaging studies of rhythm perception and reproduction consistently highlight the activation of motor areas (e.g. premotor cortex, supplementary motor area, cerebellum, and the basal ganglia) in the brain (Bengtsson, 2009; Grahn \& Brett, 2007). A fMRI study on rhythm memory also highlights the activation of the supplementary motor areas and the cerebellum, alongside activation of the inferior frontal gyri and the inferior parietal lobules (Kanoike et al., 2012). A PET study by Jerde et al., 2011 compared working memory for rhythms and pitches in non-musicians and found overlapping specialized systems in the neural pathways in both domains. Unique areas that were activated during rhythm working memory (detecting a target rhythm in 10 probe rhythm sequences) were the cerebellar hemispheres and vermis (bilateral), the left anterior cingulate gyrus as well as the right anterior insular cortex. Overlapping activation for rhythm and pitch memory was found in the right inferior frontal gyrus and the left anterior cingulate cortex (Jerde et al., 2011).

Research in memory tasks looking at other auditory domains such as short-term memory for pitches (Williamson, Badderly \& Hitch, 2010), working memory for musical chords (Pallesen et al., 2010) and musical tones (Schulze, Müller \& Koelsch, 2011) have shown that highly trained musicians outperform non-musicians and suggest that musicians revert to more pronounced and reliable mechanism in the auditory memory process of musical material, which may have been developed with their many years of musical training and the requirement to memorize hours of auditory and motor information. Furthermore studies have shown that musicians as well as children after one year of musical training also show superior verbal memory performance, but only when the stimuli where presented aurally. When verbal stimuli were presented visually no differences were found between musicians and participants without musical training (Tierney et al., 2008; Ho et al., 2003) showing that superior memory in musicians is linked to the auditory modality in general. 
To be able to systematically evaluate the memory capacity of musical rhythms, a rhythm span task was developed following the established pitch memory span task by Williamson and Stewart (2010). This procedural similarity should allow a direct comparison of short-term memory for rhythms and other memory domains such as pitch memory and verbal memory (e.g. digit span) to compare memory processes and underlying neural pathways in different domains.

In the rhythm span task subjects are asked to make same/different judgments after hearing two rhythm sequences belonging to one trial. The sequences of the rhythm span task increase and decrease in length according to the participants' performance and therefore the task difficulty adapts to individual demands and memory limits and measures rhythm memory capacity (i.e. how many rhythm elements and what length of rhythm sequences people can hold in memory). The present study compares performance on this task between two groups (non-musically trained people and highly trained musicians) to see whether musical training influences performance. It was hypothesized that musicians will show better performance than non-musicians (Wallentin et al., 2010). The pitch memory span task (Williamson \& Stewart, 2010) was also included in order to show that musicians also outperform musically untrained subjects on the pitch memory span task (as superior musicians' performance on pitch memory tasks was shown by Williamson et al., 2010). Furthermore the aim was to look at how performance in the two comparable span tasks (rhythm and pitch) relate to each other. Additionally the self-report questionnaire of the Goldsmiths Musical Sophistication Index (Gold-MSI; Müllensiefen et al., 2014) was used to evaluate musical behaviour and training.

\section{Methods}

\subsection{Participants:}

40 non-musicians and 37 musicians took part in the present study. The group of nonmusicians (less than 2 years of musical training in the past, not playing an instrument at present) consisted of 13 men and 27 women with a mean age of 23.1 years (SD: 4.0, range: 18-36). The musicians group was formed of 15 men and 22 women with a similar mean age of 23.4 (SD: 3.3, range: 18-31). Musicians were all students at a music-college with more than 10 years of musical training and all highly active music performers at present. Six string 
players, 13 wind players, eight singers, eight pianists and three musicians playing a plucked instrument comprised the musicians group (drummers were not included). To evaluate musical training we used the subscale "musical training" of the Gold-MSI questionnaire (Müllensiefen et al., 2014, see Materials for more information). The scores of the musical training dimension showed a significant difference of musical training for the two groups, $t(75)=-27.41, p<.001$, with mean group scores of 41.78 for the musicians and 14.38 for the non-musician (available score 7-49). All participants gave their informed written consent to participate in the study.

\subsection{Material:}

The rhythm memory span task was developed following the task parameters as close as possible from the established pitch memory span task (Williamson and Stewart, 2010). Six rhythmical elements were created with the program "Wavelab 5" by Steinberg. Each element was 1 second long, spanning over one quarter note (at a tempo of 60 beats a minute). All rhythmical elements consisted of one to three tones and were made of quarter notes, eighth notes, sixteenth notes and eighth note triplets (see figure 1 for the six rhythm elements), which were all presented on the same pitch $(\mathrm{A} 4=440 \mathrm{~Hz})$ with amplitude envelopes having 5 millisecond attack and decay ramps. The rhythm elements were kept simple and basic to ensure that the task was manageable. Additionally a 3-second-long mask was created out of an arbitrary irregular rhythm underlayed with white noise. The task was programmed in "Presentation" and presented over stereo headphones. At the beginning of the task sequences were made out of two rhythm elements, which were either the same or different. As the precise timing is highly important for the rhythm elements in order to make them as distinct as possible, 20 pairs of sequences (10 same and 10 different trials) were created for increasing sequence lengths ( 2 to 10 elements). Rhythm elements were randomly sampled and counterbalanced as far as possible. Furthermore, it was taken into account that sequences containing the same element length had roughly the same number of presented tones (e.g. elements with one, two and three tones were well-balanced).

The pitch span task (Williamson and Stewart, 2010) was also included to compare performance between the two memory span tasks. The musical stimuli for the pitch memory span task were formed of 10 triangle-waveform tones (equally tempered, whole tone steps) with fundamental pitches ranging from $262 \mathrm{~Hz}$ (C4) to $741 \mathrm{~Hz}$ (F\#5). Tones were $500 \mathrm{~ms}$ 
long and there was a $383 \mathrm{~ms}$ pause between them. The tones were randomly sampled to create the pitch sequences, which were two tones long at the beginning of the task. According to participants' performance sequence length increased or decreased by one tone. A trial was formed of a pair of sequences with same tone length. The task was to make same/different judgments (see Williamson \& Stewart, 2010 for more information).

To evaluate musical training and musical sophistication, the German version of the self-report questionnaire of the Goldsmiths Musical Sophistication Index v1.0 (Gold-MSI, Müllensiefen et al., 2014) was used. The questionnaire consists of 38 statements about musical engagement and behaviour and participants rated these on a seven-point scale from "completely disagree" to "completely agree". The questionnaire comprises a general factor "Musical Sophistication" as well as 5 individual dimensions: Active Engagement, Perceptual Abilities, Musical Training, Emotions and Singing Abilities. The scores range from 9-63 for Active Engagement and Perceptual Abilities, 7-49 for Musical Training and Singing Abilities and 6-42 for Emotions.

\subsection{Procedure:}

All participants completed the pitch span memory task as well as the rhythm memory span task and filled in the Gold-MSI questionnaire. Subjects were seated in front of a computer and received instructions on the screen.

For the pitch memory span tasks, two sequences with the same number of tones were played, tones were randomly sampled and the participants had to decide whether the sequences were the same or different. The participants listened to the stimuli via headphones. The experiment was double-blind to avoid bias. The researcher pressed the spacebar to start the trials. After a $500 \mathrm{~ms}$ pause the first pair of sequences was played with an inter-sequence pause of two seconds. Sequences were two tones long to begin with and the sequence length increased by one tone when the participant gave two right answers and decreased again after one wrong answer. The sequence length was determined by a two-up, one-down staircase procedure. The two sequences belonging to one trial were either identical (same tones, same order) or varied as two tones of the sequence were in the reversed position. First and last tones of a sequence remained the same (not for the two and three tone sequences). The participant gave their same/different response by saying it out loud and the researcher entered it into the computer. After a two second long pink noise burst to minimize carry-over effects and to 
clear the aural sense, the next trial was played. The procedure and task was complete when the participant's responses contained eight reversals. The span performance was calculated by considering the sequence lengths of the last six reversals.

The procedure of the rhythm span memory task was modeled after the pitch memory span. In a trial participants heard two rhythm sequences with the same number of rhythm elements and had to indicate via pressing a button if they thought the sequences were same (left command button) or different (right command button). The participant pressed the spacebar to start the test. The first pair of sequences was played and each sequence was two elements ( 2 seconds) long. There was an inter-sequence pause of two seconds. The two sequences were either identical (same elements, same order) or differed by reversed position of two rhythm elements. For sequences longer than three elements the first and last item remained the same. Participants then gave their answer by either pressing the left (same) or right (different) command button. Then the three second long mask was played to minimize carry-over effects and the participants then started the next trial by pressing the spacebar. Rhythm sequences increased and decreased according to the participants' performance by using a two-up, one down staircase procedure. When participants gave two right answers a one element longer sequence trial was played. Sequence length decreased by one element when a wrong answer was given. Eight reversals were needed to complete the task. The span score of the last six reversals were taken into consideration to calculate the rhythm span score. The first two reversals were excluded as they were considered as practice trials.

At the end of the session, participants filled in the self-report Gold-MSI questionnaire to evaluate musical training and behaviour.

\section{Results}

For the analysis two non-musicians were excluded. One participant performed more than three standard deviations away from the mean score in the pitch span task and one subject scored more than three standard deviations away on the rhythm span task. The scores of the rhythm span memory task were not normally distributed (Kolmogorov-Smirnov test reveals $\mathrm{p}<.05)$, so the non-parametric Mann-Whitney Test was used to compare group performance of both span tasks (a consistent test-method was applied to make results more comparable). To see whether the scores of the two memory tasks correlate with each other 
and with the five dimensions of the Gold-MSI questionnaire non-parametric Spearmans Correlations were applied. Table 1 gives an overview of scores for both groups for the rhythm span task, pitch span task as well as for the five dimensions of the Gold-MSI questionnaire.

Figure 2 shows the distribution of performance for the two groups, musicians and nonmusicians, on the rhythm span memory task. In order to compare group performance on the rhythm span task a Mann-Whitney test was applied and shows a significant difference between musicians (Median: 4.5) and non-musicians (Median: 3.83), $U=384.00, z=-3.39, p$ $=.001, r=-.39$. The musicians perform significantly better than the non-musicians on the rhythm span task. For the scores of the pitch memory span task (figure 3) a Mann-Whitney test also reveals a significant result: $\mathrm{U}=179.00, \mathrm{z}=-5.57, p<.001, r=.64$. The musicians group (Median: 7.50) outperformed the non-musicians group (Median: 5.75) significantly (figure 4).

Spearman's Correlations reveal that performance on the two memory tasks highly correlate, $p<.001$ (figure 5) and that both tasks also correlate with all subscales of the GoldMSI questionnaire (all $p$-values < .05). Performance on the rhythm span task was positively linked to performance on the pitch memory task and subjects' musical sophistication was also positively correlated with task performance in rhythm and pitch memory span tasks.

\section{Discussion}

The study introduces a rhythm memory task, which adapts difficulty to participants' performance and measures individual memory load capacity for rhythms. The results of the rhythm memory span task show that the task is sensitive to individual differences and confirms that experts in this domain, e.g. musicians, perform significantly better than nonmusicians. The task measures individual memory capacity for rhythmical information, which is positively correlated with the ability on the pitch memory span task as well as the five dimensions of the Gold-MSI questionnaire. Participants with higher scores in Active Engagement, Perceptual Abilities, Emotions, Singing Abilities and Musical Training perform better in the rhythm memory task.

The fact that musicians outperformed the non-musicians on the rhythm memory span task confirms our hypothesis and is in accordance with other studies showing superior performance of musicians in rhythm memory tasks (Wallentin et al., 2010). The present data 
also supports previous findings indicating superior performance of musicians in other musical memory tasks (Pallesen et al., 2010; Schulze et al., 2011) by showing that musicians also outperformed the non-musicians group on the pitch memory span task. The significant correlation of performance on the rhythm and pitch memory span task shows a strong correlation between these two memory processes. In this context, it is worth noting that the use of memory strategies is an important factor when looking at performance differences in memory tasks (Akiva-Kabiri et al., 2009; Gobet et al., 2001; Schulze et al., 2011). The musicians from the present study were mostly music students at a music college and it is part of the music theory education to be trained in musical memory strategies. The use of efficient strategies is an interesting approach for future studies in order to explore whether nonmusicians could improve musical memory performance when instructed to use specific memory strategies, such as for example cognitive chunking which has been shown to be an efficient strategy in musicians (Pike \& Carter, 2010)

Alongside highlighting activation of unique neural networks for pitch and rhythm memory processes, Jerde at al., 2012 also report overlapping brain activation in the right inferior frontal gyrus and left anterior cingulate cortex. It can be proposed that these areas play a general role in auditory memory and that a superior functional activation of these area lead to better task performance in rhythm and pitch memory. Especially the activation of the latter mentioned right anterior cingulate cortex has been reported in attention and working memory studies (Pardo, 1990) and a study comparing auditory working memory comparing musicians and non-musicians has shown greater BOLD signal in the anterior cingulate cortex in musicians (Pallesen et al., 2010). Building on these findings, better performance of musicians on both memory tasks could be due to anatomical and functional differences of the musicians' brains, which have been studied extensively (see Herholz \& Zatorre, 2012 for an overview).

The Gold-MSI questionnaire was included to not only evaluate musical training but more broadly, musical sophistication, in order to strengthen the idea of "being musical" is not only related to active musical training, but also other musical abilities. These include listening and music perception skills, as well as engaging with music through other activities (e.g. dancing to music, attending concerts, singing along to the radio) as it has been flagged up recently (Chin \& Rickard, 2012; Müllensiefen et al., 2014). The results confirm that not only musical training but also other facets of musical engagement influence rhythm and pitch memory performance. 
To date, there is no uniform position regarding how far melodic and rhythmical components define the perception of musical sequences. The dynamic model considers that rhythm and pitch are two integrative parts (Jones \& Boltz, 1989) whereas the independence model argues that rhythm and pitch are processed separately at the initial stages of musical processing (Palmer \& Krumhansl, 1987). Overall studies have shown that pitch information is more salient and that participants are more sensitive to pitch changes. This may be due to the distinctiveness of pitches as compared to rhythms in Western music, which is normatively comprised of eleven different pitch classes and a simpler set of rhythmic elements usually combining two to three durations (Hebert \& Peretz, 1997; Prince \& Pfordresher, 2012). This fact could explain superior performance on the pitch memory span (Medians of 7.5 for the musicians and 5.75 for the non-musicians) in comparison to the rhythm span (4.5 for the musicians and 3.83 for the non-musicians). Furthermore it is worth mentioning that the pitch elements where slightly shorter (883 milliseconds) than the rhythm elements ( 1 second). Another explanation for the shorter span performance in the rhythm memory task could be that the rhythm elements contained up to three units (e.g. an eight-note and two sixteen-notes) which requires higher memory effort. In this context it is also worth mentioning that to recognize the pitch the onset of the note is sufficient whereas the perception of a duration of the rhythm elements requires two onsets to be processed. The important result of this study is that performance on rhythm and pitch span correlate indicating that similar memory processes are applied and developed through musical training.

An interesting implication for future experiments would be to investigate how the inclusion of pitch information in a rhythm memory tasks, as well as adding rhythm patterns to a pitch memory task, would influence memory performances. It would be informative to explore how these two musical features influence each other and whether rhythm relies more on pitch information, as this allows the listener to organize the sequences because of periodical stress or vice versa.

Even though the results show superior performance by musicians on the rhythm memory task, further research needs to clarify whether performance differences can be traced back to better and faster perceptual abilities as shown in EEG studies (Rüsseler et al., 2001; Ungan et al., 2013) and behaviourally (Rammsayer \& Altenmüller, 2006; Geiser et al., 2009) or advanced auditory memory strategies and abilities (Berti, et al., 2006; Pallesen et al., 2010; Wallentin et al., 2010) in the process of memorizing rhythms.

Furthermore it would be very interesting to compare performance of the rhythm memory span task with other span tasks such as digit span (aurally and visually) or the Corsi 
Block Task (measuring spatial memory span) to see whether memory abilities are domain specific (e.g. auditory memory). If the rhythm span correlates with an auditory digit span task, this would seek further insight into the phonological loop involvement (Baddeley, 1990), especially of the component of timing control (Hitch et al., 1996) in the process of rhythm memory and auditory storage. Supporting this idea Saito (2001) has previously shown a correlation of auditory digit span and a rhythm memory and reproduction task. However, it would be more appropriate to compare two procedurally similar span tasks. Additionally, by comparing span tasks of different domains (e.g. auditory, visual, spatial etc.) within musicians and non-musicians could broaden our knowledge about whether musicians only dispose superior memory performance in the trained auditory domain or whether they can transfer their memory strategies to other domains.

The rhythmic sequences used in the present study followed a classical "Western" meter (i.e. duple or triple meter; London, 2004). Both musicians and non-musicians, are most familiar with this kind of material through listening to music. Additionally, the musicians have particular perceptual and motor experience with the rhythm material, as they comprehend and execute them while practicing their instrument and performing. It would be interesting for future studies to test whether the musicians' memory advantage remained the same when rhythms following less familiar, irregular meters are used. An alternative approach could also be to investigate cultural differences on this rhythm span memory task. Several studies have shown cultural differences in rhythmic perception tasks, indicating cultural influences on music cognition that are developed through listening and perceptual musical experiences which vary in different cultures (Hannon and Trehub, 2005; Hannon et al, 2012). For example, the study by Hannon et al. (2012) showed that Turkish listeners performed comparable when detecting disruptions on a simple and complex rhythm task whereas American listeners performed less accurate on the complex compared to the simple rhythm task. The results are explained by the fact that Turkish music makes use of complex rhythmical patterns whereas American music mostly relies on simple rhythms. This study implicates that culture-specific listening experience influences rhythmic pattern perception. To our knowledge cultural differences on musical memory tasks have not been studied yet.

To investigate neural correlates of the rhythm memory process it could be desirable to use non-invasive brain stimulation to test causal involvements of certain brain areas which have been shown to be active during rhythm tasks (e.g. the right inferior frontal gyrus (Jerde et al, 2012), the inferior parietal lobe (Kanoike et al., 2012) or the supplementary motor area (Bengtsson et al., 2009)). A study by our group (Schaal et al., In Press) has shown that 
performance on the pitch memory span task (Williamson \& Stewart, 2010) could be improved significantly (no improvement was found on a visual control task), after anodal transcranial direct current stimulation over the left supramarginal gyrus (part of the inferior parietal lobe), a crucial brain area of the pitch memory process. For the rhythm memory span tasks, it would be interesting to see whether stimulation of motor areas (e.g. supplementary motor area) would modulate performance on the rhythm span memory task, indicating that perceptual abilities of rhythms could play a key role in task demands or whether stimulation of parietal and frontal areas (which are more linked to memory processes) would influence task performance.

In conclusion, the study introduces a rhythm memory span task, which measures individual memory capacity for musical rhythms. Our data show that performance on the rhythm span task is superior in the musicians group and positively correlated to pitch memory span performance as well as musical engagement and training. Musicians performed significantly better on the rhythm memory span task and the pitch span task, and it would be interesting for future research to investigate if superior span performance is limited to musical materials, the auditory domain or expands to superior memory abilities in general.

\section{Acknowledgements}

We would like to thank Sven Thönes for creating the sequences for the rhythm span task. MJB was supported by the British Academy (pf100123) and is currently supported by the ESRC (ES/K00882X/1). 


\section{References}

Akiva-Kabiri, L., Vecchi, T., Granot, R., Basso, D., \& Schön, D. (2009). Memory for tonal pitches: A music-length effect hypothesis. Annals of the New York Academy of Sciences, 1169, 266-269.

Bergetsson, S., Ullèn, F., Henrik Ehrsson, H., Hashimoto T., Kito, T., Naito, E., Forssberg, H. $\&$ Sadato, N. (2009). Listening to rhythms activates motor and premotor cortices. Cortex, 45(1), 62-71.

Berti, S., Münzer, S., Schröger, E. \& Pechmann, T. (2006). Different interference effects in musicians and a control group. Experimental Psychology, 53(2), 111-116.

Chin, T.-C. \& Rickard, N. S. (2010). The Music Use (MUSE ) Questionnaire: An Instrument to Measure Engagement in Music. Music Perception: An Interdisciplinary Journal, 29(4), 429-446.

Drake, C. (1993). Reproduction of musical rhythms by children, adult musicians, and adult nonmusicians. Perception \& Psychophysics, 53(1), 25-33.

Drake, C., Penel, A. \& Bigand, E. (2000). Tapping in time with mechanically and expressively performed music. Music Perception, 18(1), 1-23.

Fischinger, T. (2011). An integrative dual-route model of rhythm perception and production. Musicae Scieniae, 15(1), 97-105.

Geiser, E., Ziegler, E., Jäncke, L. \& Meyer, M. (2009). Early electrophysiological correlates of meter and rhythm processing in music perception. Cortex, 45, 93-102.

Gobet, F., Lane, P.C., Croker, S., Cheng, P.C., Jones, G., Oliver, I. \& Pine, J.M. (2001) Chunking mechanisms in human learning. Trends in Cognitive Sciences, 5, 236-243.

Grahn, J. A. (2012). Neural mechanisms of rhythm perception: Current findings and future perspectives. Topics in Cognitive Science, 4, 585-606.

Grahn, J. A. \& Brett, M. (2007). Rhythm perception in motor areas of the brain. Journal of Cognitive Neuroscience, 19(5), 893-906.

Grahn, J. A. \& Rowe, J. B. (2009). Feeling the beat: Premotor and striatal interactions in musicians and nonmusicians during beat perception. The Journal of Neuroscience, 29(23), 7540-7548.

Hannon, E. E., Soley, G. \& Ullal, S. (2012). Familiarity Overrides Complexity in Rhythm Perception: A Cross-Cultural Comparison of American and Turkish Listeners. Journal of Experimental Psychology - Human Perception and Performance, 38(3), 543-548.

Hannon, E. E., \& Trehub, S. E. (2005). Metrical categories in infancy and adulthood. Psychological Science, 16, 48 -55.

Hèbert, S. \& Peretz, I. (1997). Recognition of music in long-term memory: Are melodic and temporal patterns equal partners?. Memory and Cognition, 25(4), 518-533. 
Herholz, S. C. \& Zatorre, R. J. (2012). Musical Training as a Framework for Brain Plasticity: Behavior, Function, and Structure. Neuron, 76(3), 486-502.

Hitch, G.J., Burgess, N., Towse, J.N., \& Culpin, V. (1996). Temporal grouping effects in immediate recall: A working memory analysis. Quarterly Journal of Experimental Psychology, 49, 116-139.

Ho, Y.-C., Cheung, M.-C. \& Chan, A. S. (2003). Music training improves verbal but not visual memory: Cross-sectional and longitudinal explorations in children. Neuropsychology, 17(3), 439-450.

Jerde, T. A., Childs, S. K., Handy, S. T., Nagode. J. C. \& Pardo, J. V. (2011). Dissociable systems of working memory for rhythm and melody. NeuroImage, 57, 1572-1579.

Jones, M. R., \& Boltz, M. (1989). Dynamic attending and responses to time. Psychological Review, 96, 459-491.

Jones, M. R. \& Yen, W. (1997). Sensitivity to time change: The role of context and skill. Journal of Experimental Psychology - Human Perception and Performance, 23(3), 693-709.

Kanoike, N., Kotozaki Y., Miyachi S., Miyauchi C. M., Yomogida Y., Akimoto Y., Kuraoka K., Sugiura M., Kawashima R. \& Nakamura K. (2012). Rhythm information represented in the fronto-parieto-cerebellar motor system. NeuroImage, 63, 328-338.

Krumhansl, C. (2000). Rhythm and pitch in music cognition. Psychological Bulletin, 126(1), 159-179.

Lerdahl, F. \& Jackendoff, R. (1983). A generative theory of tonal music. Cambridge, MA: MIT Press.

London, J. (2004). Hearing in Time: Psychological aspects of musical meter. New York, NY: Oxford University Press.

McAuley, J. D. \& Henry, M. J. (2010). Modality effects in rhythm processing: Auditory encoding of visual rhythms is neither obligatory nor automatic. Attention Perception \& Psychoacoustics, 72(5), 1377-1389.

Müllensiefen, D., Gingras, B., Musil, J. \& Stewart, L. (2014, under review). The musicality of non-musicians: An index for assessing musical sophistication in the general population. PLoS ONE, 9(2): e89642.

Nazzi, T., Bertoncini, J. \& Mehler, J. (1998). Language discrimination by newborns: Toward an understanding of the role of rhythm. Journal of Experimental PsychologyHuman Perception and Performance, 24(3), 756-766.

Pallesen, K. J., Brattico, E., Bailey, C. J., Korvenoja, A., Koivisto, J., Gjedde, A. \& Carlson, S. (2010). Cognitive control in auditory memory is enhanced in musicinas. PLOS ONE, 5(6), e11120.

Palmer, C. \& Krumhansl, C. (1987). Independent temporal and pitch structures in 
determination of musical phrases. Journal of Experimental Psychology: Human Perception \& Performance, 13, 116-126.

Pardo, J.V., Pardo, P.J., Janer, K.W., Raichle, M.E., 1990. The anterior cingulate cortex mediates processing selection in the Stroop attentional conflict paradigm. Proceedings of the National Academy of Sciences of the United States of America, 87(1), 256-259.

Pike, P. D. \& Carter, R. (2010). Employing cognitive chunking techniques to enhance sightreading performance of undergraduate group-piano students. International Journal of Music Education, 28, 231-246.

Povel, D.-J. (1984). A theoretical framework for rhythm perception. Psychological Reseach, 45, 315-337.

Povel, D.-J. \& Essens, P. (1985). Perception of temporal patterns. Music Perception, 2(4), 411-440.

Prince, J. B. \& Pfordresher, P. Q. (2012). The role of pitch and temporal diversity in the perception of musical sequences. Acta Psychological, 141, 184-198.

Rammsayer, T. \& Altenmüller, E. (2006). Temporal information processing in musicians and nonmusicians. Music Perception, 24(1), 37-48.

Rammsayer, T., Büttken, F. \& Altenmüller, E. (2012). Musicians do better than nonmusicians in both auditory and visual timing tasks. Music Perception, 30(1), 8596.

Ramus, F., Nespor, M. \& Mehler, J. (1999). Correlates of linguistic rhythm in the speech signal. Cognition, 73(3), 265-292.

Rüsseler, J., Altenmüller, E., Nager, W., Kohlmetz, C. \& Münte, T. F. (2001). Event-related brain potentials to sound omissions differ in musicians and non-musicians.

Neuroscience Letters, 308, 33-36.

Saito, S. (2001). The phonological loop and memory for rhythms: An individual differences approach. Memory, 9 (4/5/6), 313-322.

Schaal, N. K., Williamson, V. J. \& Banissy M. J. (In Press). Anodal transcranial direct current stimulation over the supramarginal gyrus facilitates pitch memory. European Journal of Neuroscience.

Schulze, K., Müller, K. \& Koelsch, S. (2011). Neural correlates of strategy use during auditory working memory in musicians and non-musicians. European Journal of Neuroscience, 33, 189-196.

Tierney, A. T., Bergeson, T. R. \& Pisoni, D. B. (2008). Effects of Early Musical Experience on Auditory Sequence Memory. Empirical Musicology Review, 3(4), 178-186.

Ungan P., Berki, T., Erbil N., Yagcioglu, S., Yüksel, M. \& Utkucal, R. (2013). Event-related potentials to changes of rhythmic unit: differences between musicians and nonmusicians. Neurological Sciences, 34(1), 25-39. 
Wallentin, M., Nielsen, A. H., Friis-Olivarius, M., Vusst, C. \& Vuust, P. (2010). The Musical Ear Test, a new reliable test for measuring musical competence. Learning and Individual Differences, 20, 188-196.

Williamson, V. J., Baddeley, A. D. \& Hitch, G. J. (2010). Musicians' and nonmusicians' short term memory for verbal and musical sequences: Comparing phonological similarity and pitch proximity. Memory \& Cognition, 38(2), 163-175.

Williamson, V. J. \& Stewart, L. (2010). Memory for pitch in congenital amusia: Beyond a fine-grained pitch discrimination problem. Memory, 18(6), 657-669.

Wu, X., Westanmo, A., Zhou, L. \& Pan, J. (2013). Serial binary interval ratios improve rhythm reproduction. Frontiers in Psychology, 4, Article 512. 


\section{Table and Figure Legends}

\section{Table 1}

Median Scores for both memory span tasks and all dimensions of the Gold-MSI questionnaire for both groups. Significant differences between groups, musicians and non-musicians, for all variables (all $\mathrm{p}$ values $\leq .001$, non-parametric Mann-Whitney Tests).

\section{Figure 1}

A The six rhythm elements from the rhythm memory span task.

B Two example trials from the rhythm memory span task for sequences with four rhythm elements. The upper two sequences are an example for a trial where sequences remain the same and the bottom two rhythm sequences represent a different trial, where bars (elements) 2 and 3 are in reversed order.

\section{Figure 2}

Scatterplot showing the score distribution of the rhythm memory span task (non-musicians: $\mathrm{N}$ $=38$; musicians $\mathrm{N}=37$ ).

\section{Figure 3}

Scatterplot showing the score distribution of the pitch memory span task (non-musicians: $\mathrm{N}=$ 38; musicians $\mathrm{N}=37$ ).

\section{Figure 4}

Median scores for both groups (musicians and non-musicians) for the rhythm span and pitch span task. Significant between-group differences ( $p$ values $\leq .001$ ) for both tasks. Musicians outperformed non-musicians in the rhythm and pitch memory span task.

\section{Figure 5}

Scatterplot showing the correlation of the scores of the rhythm and pitch memory span tasks $(\mathrm{r}=.47, \mathrm{p}<.001)$. 
Table 1

\begin{tabular}{llllllll}
\hline Group & $\begin{array}{l}\text { Rhythm } \\
\text { Span Score }\end{array}$ & $\begin{array}{l}\text { Pitch Span } \\
\text { Score }\end{array}$ & $\begin{array}{l}\text { Musical } \\
\text { Training }\end{array}$ & $\begin{array}{l}\text { Active } \\
\text { Engagement }\end{array}$ & $\begin{array}{l}\text { Perceptual } \\
\text { Abilities }\end{array}$ & Emotions & $\begin{array}{l}\text { Singing } \\
\text { Abilities }\end{array}$ \\
\hline non-musicians & 3.83 & 5.75 & 15.00 & 27.00 & 42.00 & 32.00 & 24.50 \\
musicians & 4.50 & 7.50 & 43.00 & 47.00 & 55.00 & 36.00 & 36.00 \\
\hline
\end{tabular}


Figure 1

(a)

(b)
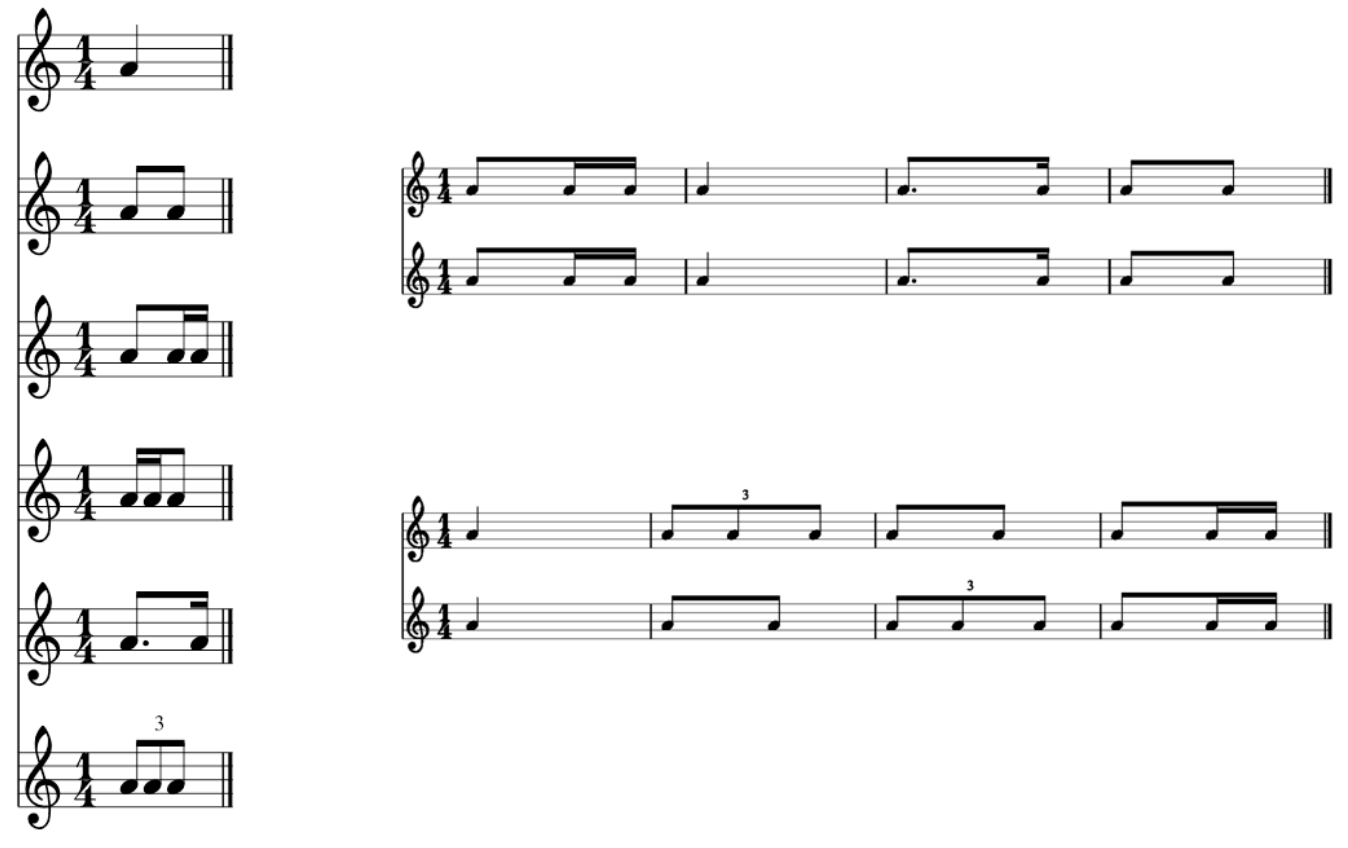
Figure 2

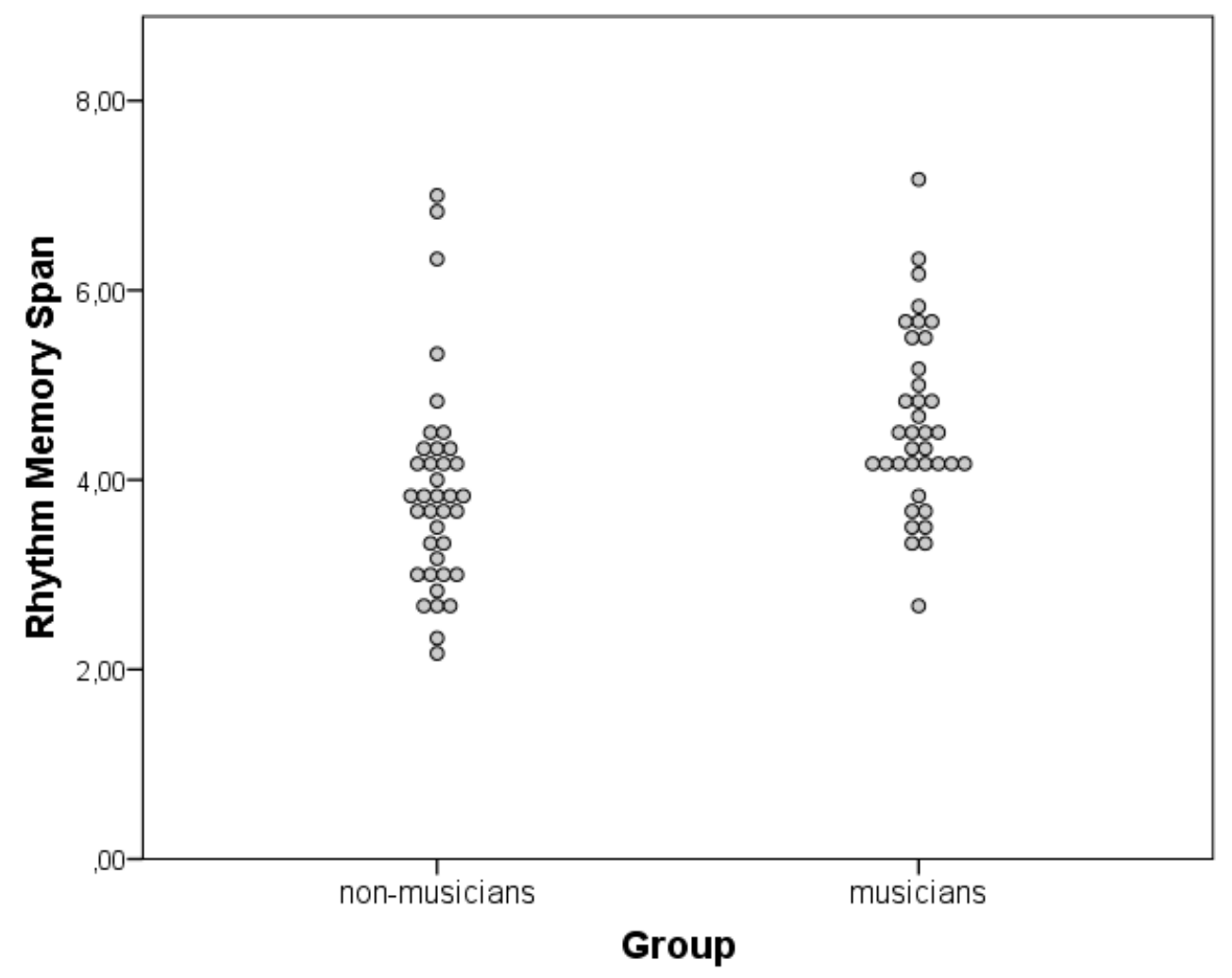


Figure 3

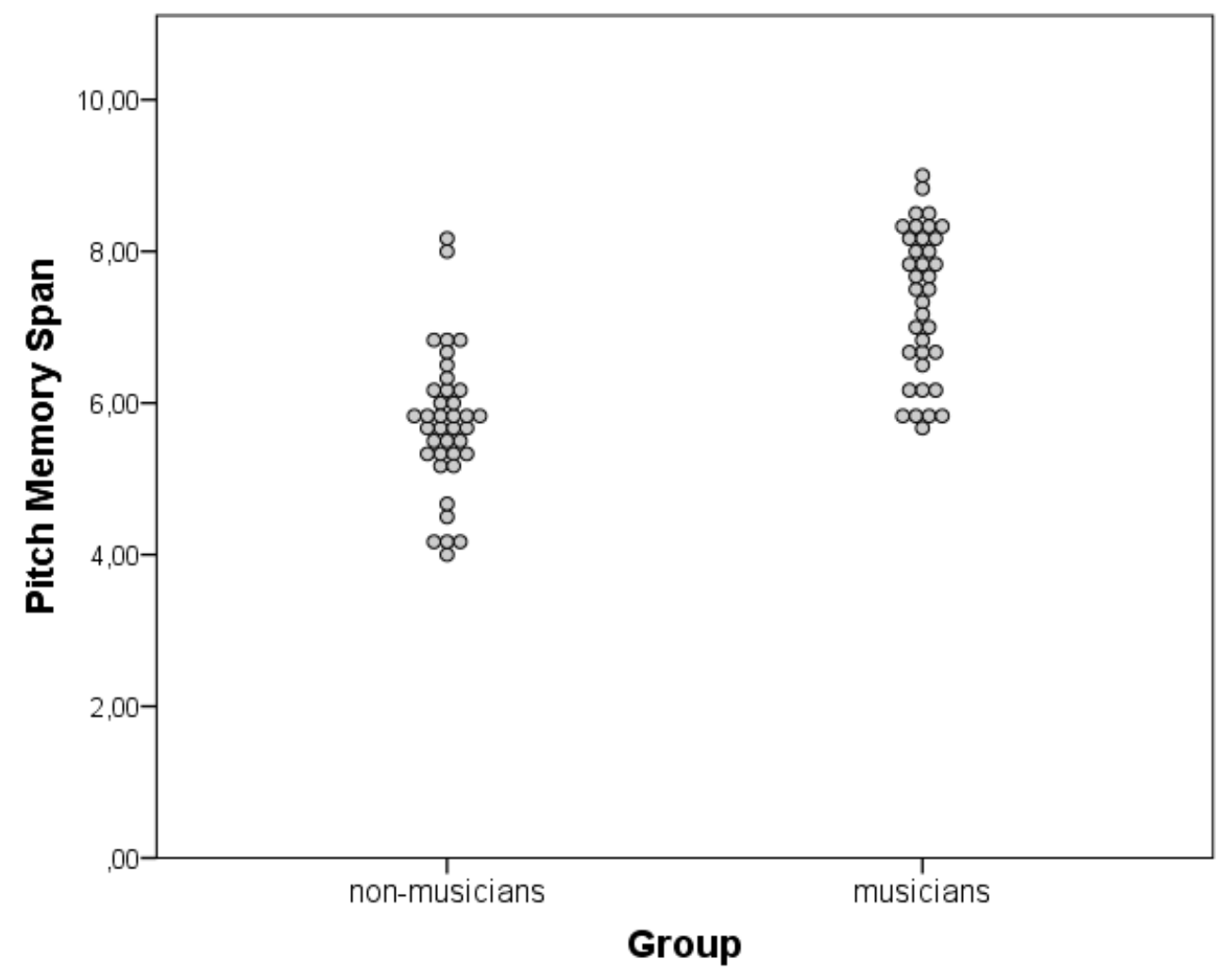


Figure 4

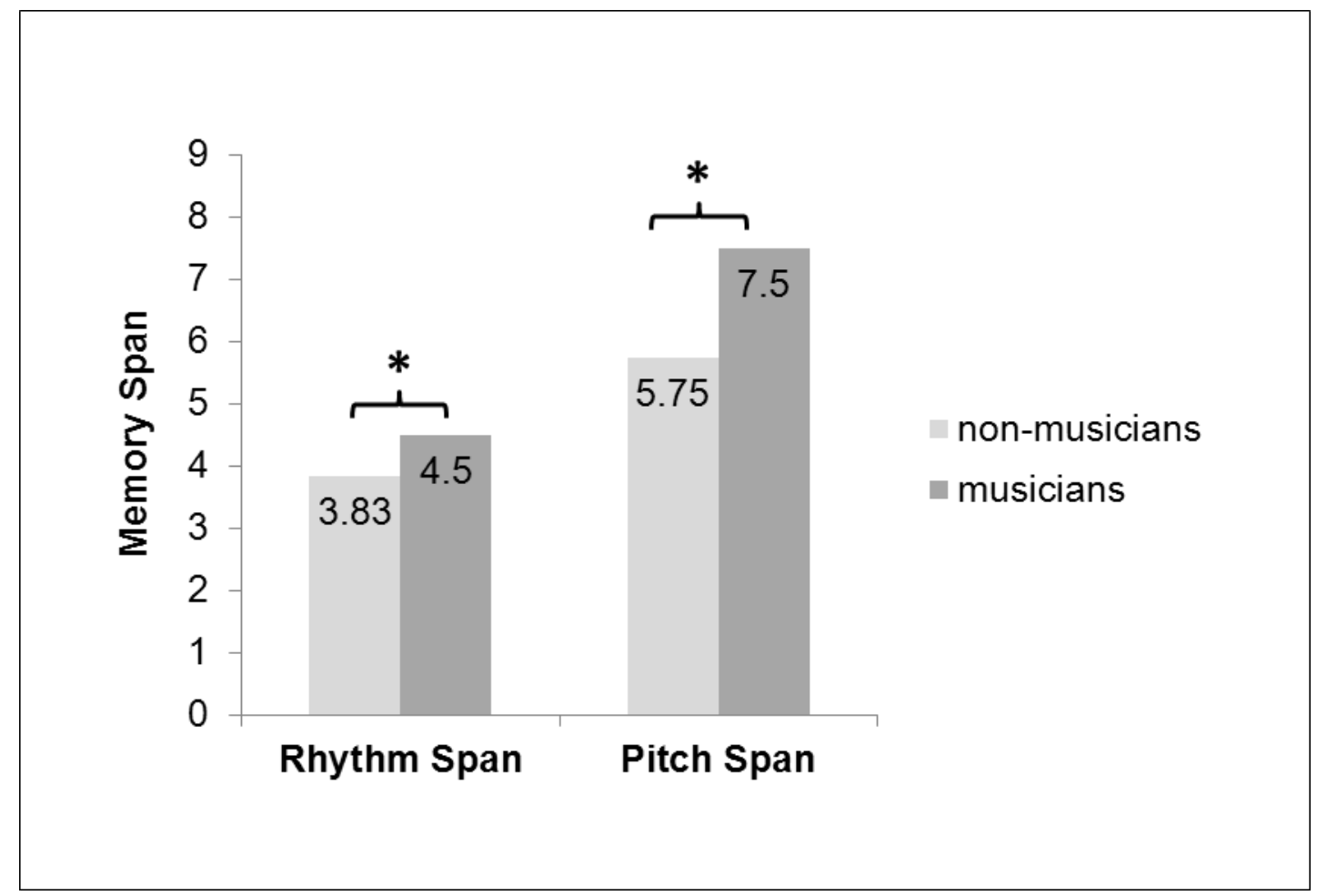


Figure 5

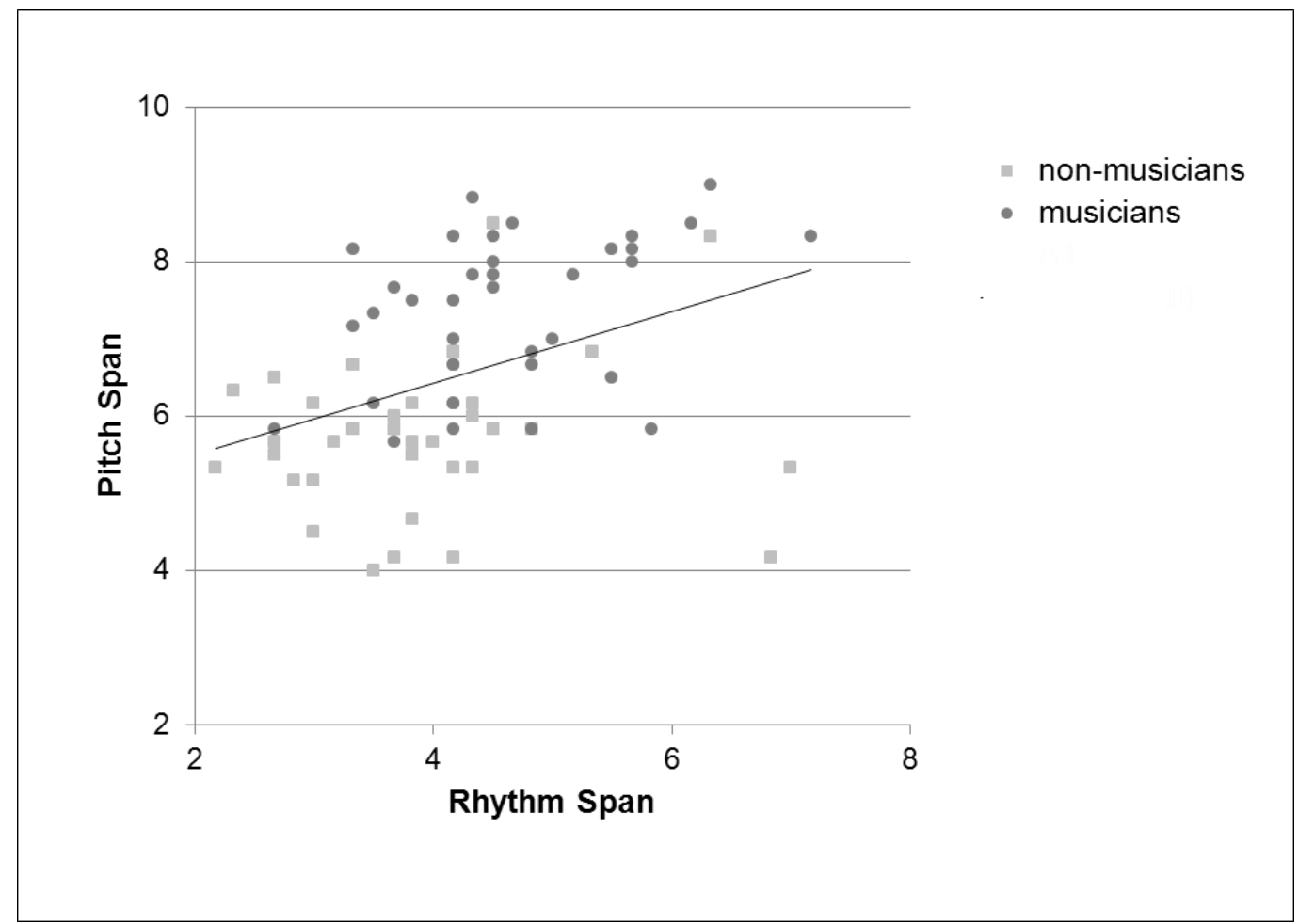

